\title{
Stable Cartels Revisited
}

\author{
EFFROSYNI DIAMANTOUdI \\ Department of Economics \\ McGill University, Montreal
}

January 2000

A THESIS SUBMITTED TO

THE FACULTY OF GRAdUATE STUdies AND RESEARCH

IN PARTIAL FULFILLMENT OF THE REQUTREMENTS

OF THE DEGREE OF

MASTER OF ART

(C) Effrosyni Diamantoudi 1999 
National Library

of Canada

Acquisitions and Bibliographic Services

305 Wetington Strees Otemen ON KIA ONA Canada
Bibliotheque nationale du Canada

Acquisitions et services bibliographiques

395, ne Welington Ottawa ON K1A ON4

Canada
The author has granted a nonexclusive licence allowing the National Library of Canada to reproduce, loan, distribute or sell copies of this thesis in microform, paper or electronic formats.

The author retains ownership of the copyright in this thesis. Neither the thesis nor substantial extracts from it may be printed or otherwise reproduced without the author's permission.
L'auteur a accordé une licence non exclusive permettant à la Bibliothèque nationale du Canada de reproduire, prêter, distribuer ou vendre des copies de cette thèse sous la forme de microfiche/film, de reproduction sur papier ou sur format électronique.

L'auteur conserve la propriété du droit d'auteur qui protège cette thèse. Ni la thèse ni des extraits substantiels de celle-ci ne doivent être imprimés ou autrement reproduits sans son antorisation. 
Contents

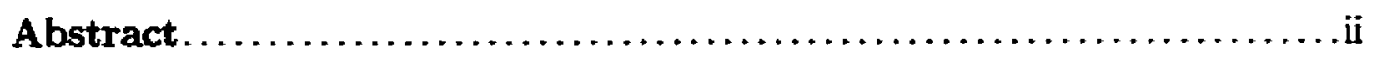

Acknowledgments $\ldots \ldots \ldots \ldots \ldots \ldots \ldots \ldots \ldots \ldots \ldots \ldots \ldots \ldots \ldots \ldots \ldots \ldots \ldots \ldots \ldots$

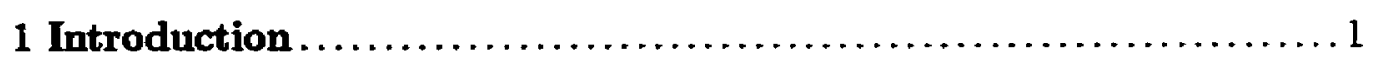

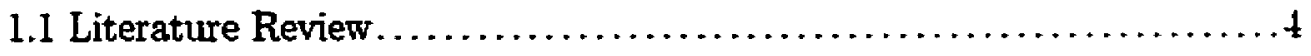

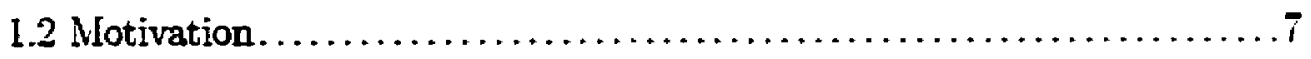

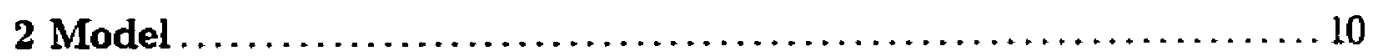

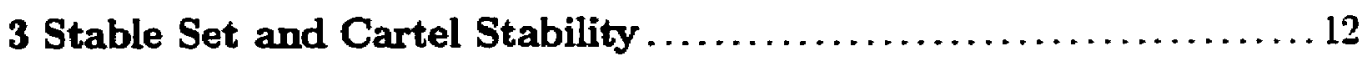

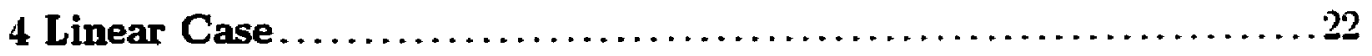

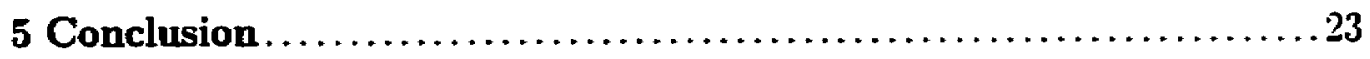

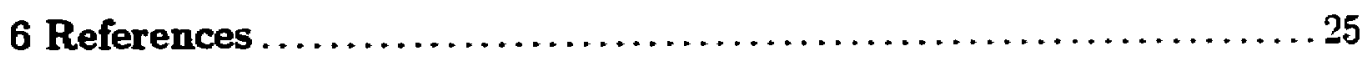




\section{Abstract}

This paper analyzes cartel stability when firms are farsighted. It studies a price leadership model as the one by $D^{\prime}$ Aspremont et al. (1983), where the dominant cartel acts as a leader by determining the market price, while the fringe behaves competitively. According to D' Aspremont et al.'s notion of cartel stability, a firm will not remain in a cartel as long as it prefers the outcome where it is the only member leaving the cartel for the fringe. Such an approach implies that the firm is myopic since it ignores whether its alternative is stable itself. Our notion captures foresight by employing a solution concept built in the spirit of von Neumann and Morgenstern (1944) stable set, yet adopting an indirect dominance à la Harsanyi $(197 t)$.

\section{Résumé}

Le mémoire étudie la stabilité d'un cartel dans le cadre d' un modèle de leadership de prix de l' entreprise dominante par D' Aspremont et al. (1983). Contrairement, notre analyse tient compte de la prévoyance parfaite des individus (entreprises) rationnels, qui n' est pas mentionée dans la littérature. Cet article contribue à la théorie de la stabilité d'une structure de marché caracterisée par la collusion des entreprise afin d' offrir une notion paralelle a celle proposée par von Neumann \& Morgenstern (1944). Néanmoins, nous captivons clairvoyance par utilise une dominance relation indirecte comme celle de Harsanyi (1974). Nous presentons également un théorèm d' existence d' un cartel dominant stable. 


\section{Acknowledgments}

Writing a thesis is a challenge to a student primarily because it is most likely one of the first times the student gets to formally expose his or her own original ideas. Fortunately, the fear of acceptance and understanding does not prevail due to the support and encouragement the student receives throughout the process by those around him.

The person to whom I am indebted the most is my supervisor Professor Joseph Greenberg. Without Yossi not only this project would not have been written. but I wouldn't even be doing my graduate studies in economic theory to start with. It is him that inspired me by exposing me to game theory, that taught me the foundations of research. It is him that taught me how to organize my thoughts into a concise idea. But most importantly, it is Yossi that was there for me as a friend, more than a supervisor, and encouraged me when nothing seemed to work.

Finally I would like to thank my husband Licun, who besides being a most supportive spouse, is also a great colleague. His constant advising and help are an integral part of this project. 


\section{Introduction}

Although the need to study and analyze cartel stability is evident from the reference to it in every industrial organization textbook and handbook, it seems that no satisfactory answer has yet been given, as it will be illustrated throughout the remaining of this work. The aim of this work is to offer another stepping stone to this direction. By using a conceptually different approach, we aspire to shed some light to the rather debatable aspects of cartel stability.

The classical lecture any economics student receives is that in an oligopolistic (finite number of firms) market even though collusive behavior, that is. all firms acting as one monopolist, is more profitable than competitive behavior, that is, each firm maximizing independently its own profits while ignoring the strategic interdependence of the environment, collusion will not prevail. The explanation supporting this prediction is that given that every one else colludes and behaves as one firm, each firm, unilaterally, has an incentive to deviate by behaving competitively by itself. The cartel would price and produce where its marginal revenue equals its marginal cost, whereas the cheating firm would function as a price taker and produce at the point where its marginal cost equals the market price, as set by the cartel.

The fact that empirical studies contradict the prediction of the above theory was one of the reasons that lead theorists to reconsider their position. The conceptual difficulties implicit in the model was another. An immense literature has developed since, studying (the possibilities of) collusion within a dynamic environment where firms interact repeatedly. Using the theoretical tools provided by the study of re- 
peated games, economists constructed different models where punishment strategies may vary, where uncertainty is introduced, where signals are employed, etc. Most of this works end up supporting some form of collusion one way or another.

But the question in an one-period environment is dealt with in much less works. By one-period environment we do not imply necessarily a situation where all firms make their decision and they cannot revise it whatsoever, that is, a classical normal form game. Instead, we refer to the situation where the profits are collected once at the end of the market clearance. Therefore dynamic models that describe the oligopolistic markets through two or more stage games (but not repeated games) are also included in this class.

One of the difficulties inherent in the one-shot approach lies in the institutional assumptions adopted by the model. In particular, is it strictly a static environment? If it is, then at which time do agents deviate? Or is it the case that "somehow" a recommendation is put on the table and they just do not follow it? But if there is time for one deviation then may be there is time for more... Or is it pseudodynamics? That is the moves, and countermoves take place in the agent's mind, whereas he literally moves only once. Questions of this sort, gave rise to a few models that took different stands with respect to the time line of the environment.

Another difficulty, stemming from the above is the solution concept the theorist employes to analyze a situation that is neither clearly static nor dynamic. Of course if one defines the oligopolistic market through some tree, or through a normal form game the answer is rather obvious. But how does one deal with pseudodynamics? Then the choice of solution concept, if there exists an appropriate one for the story the modeler is trying to tell may become cumbersome, and a constant source of 
controversy.

Lastly, do firms behave non-cooperatively, or do they cooperate? It is an oxymoron scheme to analyze collusion in a non-cooperative manner, yet, the distinction in the literature of oligopolistic markets is drawn on binding agreements. If binding agreements are not institutionally permitted, which is the case in the real world through antitrust policies, for a cartel to survive it must be immune to deviations. The agent must want to stick to the agreement since he cannot be forced to. An agent is assumed to want something when he has no other strictly better alternative. Although it may seem that we are alluding that the non-cooperative approach better describes the oligopolistic markets, it is not our intention and the following argument will clarify it. Indeed. for a cartel to survive it has to be immune to deviations. What we didn't fully and formally specify yet is a deviation. The non-cooperative approach focuses on individual deviation, whereas a cooperative approach allows for more than one players to deviate at a time. In fact, if our goal is to better describe what really goes on in the real world, coalitional deviations are far more natural than individual ones, as long as we compare individual payoffs of coalition members versus the aggregate profits of a coalition. The cooperative approach has contributed considerably to the study of cartel stability by treating the different cartels as coalition structures and thus applying solution concepts appropriate for cooperative games. Nevertheless. such an approach is not immune to criticism either: by defining a situation through a game in coalitional form, binding agreements are implicitly assumed. We believe that this is not true, instead the level of commitment depends on the solution concept one employs to a cooperative game. Then we do admit that indeed most cooperative solution concepts do rely on binding agreements. 
The venue we follow in this project is to assume that binding agreements are not possible, and therefore examine the immunity of cartels against potential deviations. We do not allow for coalitions to form, apart from the cartel itself. However, unlike the classical non-cooperative approach when we examine the desirability of a deviation by a single agent we do not assume that every other agent will stick to his original plan, regardless of its profitability.

The works that studied cartel stability within a static environment using a noncooperative approach are presented in the next subsection, followed by a trivial example that illustrates the need for our contribution.

The next section presents formally our model, whereas the third section discusses the solution concept we use to solve our model, as well as its existence. The fourth section demonstrates the results of our general model in the specific case of linear demand and marginal cost functions. Finally, the last section concludes our work.

\subsection{Literature Review}

The most influential work done on cartel stability is admittedly the one by $D^{\prime}$ Aspremont et al. (1983). Their model based on a more general price-leadership framework, considered a finite economy where a dominant cartel sets the market price at a joint profit maximizing level, and a competitive fringe free-rides on the profit maximizing efforts of the cartel by violating the quota — producing more- set by the cartel.

Although, price-leadership models were studied in earlier works and not necessarily in an attempt to study cartel behavior, the major contribution of ' $D^{\prime}$ Aspremont et al. (1983) was the observation that once a member of the cartel deviates and joins the fringe, the cartel is going to adjust its behavior! An implicit assumption, that may be bothersome to those concerned with the applicability of the model, is the 
perfect monitoring. If a firm deviates from the cartel and joins the fringe its move will be noticed by the cartel and taken into consideration accordingly. Precisely this consideration will lead to the adjustment of their quota (and by extension of the market price) in a manner that maximizes the new aggregate cartel profits.

Once such an adjustment is captured by the model, the outcome is that it is not always beneficial for a firm to exit the cartel and join the fringe. The profits the potential deviant may enjoy by increasing his output may be offset by the decrease in market price as brought about by the cartel adjustment. In particular, the authors formally show that there always exists a stable cartel. That is, a specific size of a cartel such that it is not profitable for a firm to violate the quota anymore and join the fringe. Moreover, no more members of the fringe wish to join the cartel either. It is also shown that the same results do not hold for the case where the economy is comprised of an infinite number of firms.

Along the same lines and almost parallelly another paper was published by Donsimoni et al. (1986) where the same general model is studied with the additional assumption of linear demand and marginal cost functions. Besides their different approach towards existence, they also show that under some additional conditions on cost efficiency the stable cartel is unique.

Within the same institutional setting other works altered some of the assumptions of the model. Specifically, Donsimoni (1985) allowed for product heterogeneity: and studied cartel stability within this more complex environment. More recently; Shaffer (1995) studied a different situation where the fringe does not behave that competitively" anymore, instead the fringe members realize the strategic impact of their actions, that is the impact their production has on residual demand and thus 
on the market price. Thus, the fringe behaves as in a Cournot competition vis a vis perfect competition.

The most recent works, those of Thoron (1998) and Prokop (1999) took a different direction. Instead of manipulating the assumptions of the model, they dug deeper into it, by trying to explicitly describe the formation of a cartel. Within a dynamic context they formalize the process of joining or leaving a cartel. The Thoron (1998) approach does not employ a classic dynamic framework, i.e., a game tree, yet it does presume two independent stages. In the first, firms decide whether to cooperate or not and in the second they determine their output. By disintegrating the decision process of a firm the author allows for different solution concepts to apply to the two separate stages. In particular. a solution concept that allows coalition formation is employed at the stage were the cartel determines its output, whereas a more noncooperative notion is used to determine the unilateral decision of a firm to join the cartel or not. Although the decomposition of the decision making process allows the theorist to deal with each part most appropriately, it may loose information by disregarding the strategic element between the two stages.

Diversely, Prokop (1999) utilizes extensive form games to describe the process of collusion. Each firm one after the other decide to enter the cartel or not, once a firm makes its choice, it commits to it, and cannot change its mind later on. The notion of subgame perfection is applied to it, and through this, the author may argue that through backward induction, a frrm can foresee what others will do in subsequent moves and act accordingly. It turns out that some firms will choose to stay outside the cartel and some will choose to enter it. Those that are in the fringe are those that moved first, whereas the last firms to move are those forming the cartel. Thus, 
those moving first are better off than those moving later on, but how is the order of moves determined? Why should the firms respect the order and not cheat on it if that will make them better of? The firm's commitment to stand by its decision is not justified and, in fact, it is recognized as a weakness by the author. It is interesting to point out though, that the Prokop dynamic approach yields the same results (stable cartels) the D' Aspremont approach does, implying perhaps, that such a commitment is implicit in the D' Aspremont model as well.

Lastly, $\operatorname{Li}(1992)$ studies an oligopoly model, that unlike the ones mentioned so far, does capture foresight in a manner similar to the one we will propose. Nonetheless. his work also assumes cooperative behavior, that is, coalitions can form and act. whithout the presumption of binding agreements. Such an institutional assumption is rather strong and unjustifiable, and therefore not part of our analysis.

\subsection{Motivation}

A common characteristic among the above cited literature is the lack of foresight on behalf of the firms. The following trivial example is meant to clearly point out the myopia embedded in the analysis of stable cartels. The firms' behavior and the various institutional assumptions are identical to those in the $D^{\prime}$ Aspremont et al. paper (1983).

Consider a market with $N=\{1, \ldots, 5\}$ identical firms - they have identical cost function - and let $Q=\sum_{i \in N} q_{i}$ indicate the total quantity produced in the market, whereas $q_{i}$ indicates the individual firm quantity. Let $P$ indicate the market price.

Consider the simple case of a linear demand where $Q=100-P$ and where the individual cost function is $T C\left(q_{i}\right)=\frac{\mathrm{L}}{2} 10 q_{i}^{2}$. When a cartel of size $k \leq n$ forms it behaves like one firm by producing and pricing at the point where the marginal 
revenue (derived from the residual demand) equals marginal cost, and the fringe $n-k$ behaves competitively by producing at the point where market price is equal to the marginal cost of each firm. The firms in the cartel are aware of the behavior of the fringe and this awareness is reflected on their perception of the marginal revenue as derived from the residual demand.

The following table illustrates the profits per firm for the 5 different market structures:

\begin{tabular}{|c|c|}
\cline { 2 - 2 } \multicolumn{1}{c|}{} & $\swarrow \pi^{f}(0)=222$ \\
\hline$\pi^{c}(1)=223$ & $\swarrow \pi^{f}(1)=224$ \\
\hline$\pi^{c}(2)=226$ & $\swarrow \pi^{f}(2)=230$ \\
\hline \hline$\pi^{c}(3)=231$ & $\pi^{f}(3)=241$ \\
\hline$\pi^{c}(4)=239 \nearrow$ & $\pi^{f}(t)=258$ \\
\hline$\pi^{c}(5)=250 \nearrow$ & \\
\hline
\end{tabular}

Where $\pi^{c}(k)$ denotes the profits of a firm belonging to a cartel of size $k$, whereas $\pi^{f}(k)$ denotes the profits of a firm belonging to the fringe of a cartel of size $k^{l}$. Note that $\pi^{f}(0)$ depicts the profits per firm of a perfectly competitive market, whereas $\pi^{c}(5)$ depicts the profits per firm of a perfectly collusive market.

According to the $D^{\prime}$ Aspremont et al. paper (1983) it is supported that only the cartel of size 3 is stable. More analytically, cartel of size 1 is not stable because members of the fringe wish to join in $(226>224)$. Similarly, cartel of size 2 is not stable since members of their fringe wish to join in $(231>230)$. Furthermore, cartel of size 5 is not stable since a member wishes to exit and join the fringe of cartel of size $4(250<258)$. Lastly, cartel of size 4 is not stable since a member wishes to join the fringe $(239<241)$. Cartel of size 3 , however, is stable since no one wants to leave $(231>230)$ and no one wants to join in $(239<241)$. Instead the point we want to make is the following: once cartel of size 5 is formed everyone is earning

${ }^{1}$ Detailed calculations of the profits can be found in section 4. 
250. Of course we know that each one individually would like to deviate, in order to earn 258. But a farsighted firm would see ahead and realize that cartel of size 4 is not a stable one, because once there one more firm would wish to deviate and join the fringe and earn 241 vs 239 . Once down to size three no more firms would like to deviate since they would loose 230 vs 231 . Therefore, when the very first firm contemplates in deviating from the first cartel of size 5, it should foresee that its final payoff would be 241 and not 258, and it should compare 241 to 250 which is not as attractive anymore!

It is apparent that the reason that cartel of size 5 is characterized as unstable is because it is "dominated" by the (fringe of) cartel of size 4 . Yet, cartel of size 4 is "dominated" as well by the (fringe of) cartel of size 3 . In essence, the perfectly collusive situation is discredited by an outcome that is itself discredited. This same line of thought lead to subgame perfection of Nash equilibria and to criticism of the core. As theorists we aught to allow agents to compare their status quo with what will really happen to them, once all reactions and counter-reactions are over. In this spirit, cartel of size 5 should not be deemed unstable!

A first reaction to our argument would be the dynamics we introduce within a static environment, but we believe that the environment was not completely static to start with. In the D' Aspremont et al. (1983) model and its variants, it is assumed that once a firm deviates the cartel will adjust the market price, according to the new residual demand, and the new aggregate marginal cost. In fact, this price adjustment and the deviating agent's ability to foresee this adjustment was the very merit of the paper. Therefore, the model implies that a physical reaction takes place after a

\footnotetext{
"We use the word "dominance" in an informal manner, just to imply that one situation is prefered to another.
} 
physical deviation. Are we to assume thus, that the model is built over two periods? If that is the case, then an explicit description of such clean dynamics is necessary where the order of moves is specified. Or should we assume, that firms can change behavior (strategy) so quickly that it essentially happens within zero time? If the latter is the case then we can easily assume that a finite number of firms can change their behavior (strategy) within the same zero time, until a status quo is reached where no more firms wish to adjust their behavior any longer. In such a "looser" approach no order of moves needs to be specified. Describing such an environment through a game tree, would be impossible. As mentioned in the previous section, some attempts have been made to model cartel formation through game trees. but then there is an implicit binding commitment by the agent whose turn past not to change his strategy anymore.

The myopia embedded in the D' Aspremont et al. (1983) is exactly the hurdle we attempt to overcome in this work. In particular we built a solution concept in the spirit of von Neumann and Morgenstern (vN-M) stable set while altering the dominance relation, which gives us a set of stable cartels that would survive credible deviations.

\section{Model}

Let the industry $N=\{1,2, \ldots, n\}$ consist of a finite number of $n$ identical firms producing a homogeneous output. The product's market demand is represented by a differentiable function $D(P) \geq 0$ such that $D^{\prime}(P)<0$, where $P$ is the market price. Each firm has the same differentiable cost function $C\left(q_{i}\right)$ with marginal cost $C^{\prime}\left(q_{i}\right)>0$ and $C^{\prime \prime}\left(q_{i}\right)>0$ where $q_{i}$ is the quantity produced by firm $i$ and $Q=\sum_{i \in N} q_{i}$ denotes 
the total quantity supplied in the market. Let $C_{k} \subset N$ indicate a cartel of size $k \in\{0,1, \ldots, n\}$ and $F_{k}=N \backslash C_{k}$ indicate the fringe of cartel $C_{k}$.

The classical price-leadership model that we will analyze assumes that $k$ firms form a cartel $C_{k}$ and the rest form a fringe $F_{k}$ of size $n-k$. The cardinality of the sets will be denoted by $\left|C_{k}\right|=k$ and $\left|F_{k}\right|=n-k$. Note that the subscript of a cartel indicates its size, whereas the subscript of a fringe indicates the size of its respective cartel.

The fringe behaves competitively by setting its marginal cost equal to the price determined by the cartel, i.e., $C^{\prime}\left(q_{i}\right)=P$. Therefore, since all firms are identical we will suppress the subscript of individual quantities. Instead we denote each fringe member's output by $q^{f}(P)$, and the total fringe supply by $Q^{f}=(n-k) q^{j}$.

The cartel $C_{k}$ is, in turn, faced with a residual demand, $R D(k, P)=D(P)-Q^{f}$. The cartel behaves like a monopolist by setting its marginal revenue with respect to the residual demand equal to its marginal cost. Such a joint profit maximization leads the cartel members to produce $q^{c}$ each, and since they are identical $Q^{c}=k q^{c}$ as a group.

Let $P(k)$ indicate the market price a cartel $C_{k}$ has chosen, and let $\pi^{c}(k)$ indicate the profits of each member of the cartel. Similarly, let $\pi^{f}(k)$ indicate the profits of each member of the fringe $F_{k}$.

We should add that if $k=0$ then $\left|F_{0}\right|=n$ and our industry is perfectly competitive, whereas if $k=n$ and $\left|F_{n}\right|=0$ every firm has joined the cartel and our industry is perfectly collusive.

Furthermore, as has already been established by $\mathrm{D}^{\prime}$ Aspremont et al. (1983) $\pi^{c}(k)<\pi^{f}(k)$ for $k>0$. However, what is of interest to the agents is not the afore 
mentioned relationship since once a firm exits the cartel and joins the fringe the size of the cartel is not $k$ anymore, but it is $k-1$ and the size of the fringe becomes $n-k+1$ accordingly, which in turn alters the residual demand function and therefore the market price $(P(k-1) \neq P(k))$ chosen by the shrunk cartel.

Previous works took this price adjustment into consideration by having a cartel member compare $\pi^{c}(k)$ to $\pi^{f}(k-1)$ when contemplating deviation, and a fringe member compare $\pi^{f}(k)$ to $\pi^{\mathfrak{c}}(k+1)$ when contemplating joining a cartel. As a result. if $\pi^{c}(k)>\pi^{f}(k-1)$ no firm would wish to exit the cartel and thus the cartel is characterized internally stable, whereas if $\pi^{f}(k)>\pi^{c}(k+1)$ no fringe member would wish to join the cartel, which is thus characterized externally stable. If a cartel $C_{k}$ is both internally and externally stable then it is called stable.

As we extensively argued in the Introduction such a behavior on behalf of the firm is myopic or partially farsighted, since it assumes that once it deviates (if it finds it profitable) it is there to stay and nothing else is going to change. This bothersome assumption has lead us to search for a different method of examining whether a cartel is stable or not that would incorporate a fully farsighted behavior on behalf of the firms.

\section{The Stable Set and Cartel Stability}

The issue of credibility and foresight has risen on several occasions in economic models and more fundamentally in solution concepts within an econornic or game theoretic context.

In particular, when agents contemplate on their choices, they have to choose from a set of options whose outcomes they either know with certainty or they have some 
(subjective) probability distribution over them. It is not acceptable for a rational agent to assign a positive probability to an event that will never happen, unless other behavioral assumption (bounded rationality, insanity, etc.) have been imposed. The lack of credibility of Nash equilibrium when used in dynamic environments paved the way to Subgame Perfect Equilibrium, and a series of other refinements that apply in different institutional settings, i.e., incomplete information, coalition formation, etc. The original Core has been criticized heavily for the lack of credibility of dominant outcomes. Namely when an outcome is excluded from the Core it is because it is dominated by some other outcome. This other dominant outcome though is not checked against its own credibility, nameiy whether it is dominated itself by some other outcome or not.

In our case when a firm contemplates exiting a cartel $C_{k}$ it compares $\pi^{c}(k)$, that is, the profits it makes while a member of the cartel, with the profits it will make once it exits and joins the fringe $F_{k-1}$, that is, $\pi^{f}(k-1)$. The firm implicitly assumes that once it deviates, no one else will want to deviate and therefore it will enjoy profits $\pi^{f}(k-1)$ with certainty. But as we argued in the Introduction this is not always the case, in fact, it is possible that another firm may wish to exit cartel $C_{k-1}$, by now. and join the fringe $F_{k-2}$, and so on. Thus, the firm should compare its status quo $\pi^{c}(k)$ with the final outcome that will result once it initiates a sequence of events by exiting $C_{k}$. This final outcome can be characterized as such only if no more firms wish to exit and no more firms wish to join if, in other words, it is stable itself. Put differently, we can determine whether a cartel is stable or not, only if we know what every other cartel is! Such a recursive approach is adopted by the classical notion of the abstract stable set. 
The (abstract) stable set originally defined by von Neumann and Morgenstern (1947) over an abstract system is a solution concept that captures consistency. In particular, consider a social environment with a set of individuals, $N$, who face a set of alternatives $Z$. For instance, let the triple $G=\left(N,\left\{Z^{i}\right\}_{i \in N},\left\{u^{i}\right\}_{i \in N}\right)$ represent a normal form game, where $N$ is the set of players and for $i \in N, Z^{i}$ is the non-empty set of strategies of player $i$, and $u^{i}$ is player $i$ 's payoff function, $u^{i}: Z^{N} \rightarrow R$. where for $S \subset N, Z^{S}$ denotes the Cartesian product of $Z^{i}$ over $i \in S$, i.e., $Z^{S}=\prod_{i \in S} Z^{i}$ : Then $Z$, the set of outcomes is such that $Z=Z^{N}$. Let $>$ be a dominance relation defined on $Z$. Note that a dominance relation can incorporate the "rules" of the game by describing more than the preferences in the conventional sense, specifically it can describe who can induce what. For $a, b \in Z, b$ is said to dominate $a$, or $b>a$, if there exists $i \in N$ such that $a^{-i}=b^{-i}$, and $u^{i}(a)<u^{i}(b)$.

A pair $(Z,>)$ is called an abstract system and it can describe a variety of complex social situations, where normal form games are a special case as illustrated above.

Definition 1 The set, $V \subset Z$ is

1. a $v N-M$ internally stable set if $V$ is free of inner contradictions. i.e. there do not exist $x, y \in V$ such that $y>x$,

2. a $v N-M$ externally stable set if $V$ accounts for every alternative it excludes, i.e., if $x \notin V$, it must be the case that there exists a $y \in V$ such that $y>x$, and

3. a vN-M (abstract) stable set if it is both $v N-M$ internally and externally stable.

In the same way we described normal form games through an abstract system we can also describe cooperative games. In such a case, $V$ is equivalent to the vN-M solution for the cooperative game. 
The stable set approach instead of characterizing each outcome independently, it characterizes a solution set, that is, a collection of outcomes that are stable, while those excluded from the solution set are unstable.

It is immediate from Definition 1 that the stable set possesses two properties, internal stability and external stability, which happen to be the very features of the stable set that constitute it a very appealing solution concept by capturing consistency. Its appeal is captured and improved upon by Greenberg (1994). In the Theory of Social Situations (TOSS), a unifying approach towards cooperative and non-cooperative game theory, where any behavioral and institutional assumptions are explicitly defined, an equivalence is shown between the vN-M stable set and the Optimistic Stable Standard of Behavior (OSSB), a solution concept built in the spirit of vN-M stability, yet with the precise assumption of optimistic behavior explicitly formalized. TOSS amplified the pertinence of stability by recasting the dominance relation into a broader concept beyond the boundaries of a binary relation. In doing so, behavioral assumption can be imposed on the agents, and more complex institutional setting can be analyzed.

It is imperative to point out that the terms internal and external stability do not imply the same features as they did in the context of cartel stability. Characterizing an abstract set as internally stable implies that no contradictions are allowed within the set, that is; no outcome that belongs to the set dominates ${ }^{3}$ another outcome that also belongs to the set. Similarly, when an abstract set is externally stable it accounts for all the outcomes it excludes, that is every outcome that does not belong to the abstract set is dominated by some outcome inside the set. Such an

${ }^{3}$ According to its formal definition within the abstract system. 
approach achieves consistency. Whichever outcome is considered stable cannot be dominated by another stable outcome, while the unstable outcomes are dominated by stable ones. Unfortunately, as the reader may have already realized there is an abuse of terminology, in the sense that internal and external stability when used in a stable set context have the above described definition, whereas when used to describe a cartel have a different meaning as defined in earlier sections. Since this paper studies the stability of cartels we will use the terms internal and external stability to characterize cartels as the rest of the cartel literature does, and not in the way vN-M stable set does, while of course keeping the features they can attribute to a solution set.

Although the stable set solves many problems common to the other solution concepts it is not itself immune to criticism. Harsanyi (197t) criticizes the vN-M solution for its failing to incorporate foresight. Such a criticism can also apply to an abstract stable set $V$ for $(Z,>)$. Farsighted players do not just look one step ahead. Recall the normal form case, although player $i$ may be able to induce $b$ from $a$ and indeed he may prefer $b$ to $a$, he should examine whether any more moves will take place (by other players perhaps) once $b$ becomes the status quo. For this reason, Harsanyi suggests to replace the dominance relation in the definition of $V$ be some tindirect dominance" relation, which captures the fact that farsighted individuals consider the final outcome that their actions may lead to. An alternative $b$ is said to indirectly dominate another alternative $a$ if $b$ can replace $a$ in a sequence of "moves", such that at each move the active player prefers (the final alternative) $b$ to the alternative he faces at that stage.

Harsanyi's criticism inspired a series of works by Chwe (1994), Xue (1998) and 
others. All these works acknowledged the merits of abstract systems and the stable set, and contributed to the effort of a more unifying (and thus general) method of describing social environments, while emphasizing the issue of foresight by building on Harsanyi's indirect dominance.

Although the notion of the stable set is very appealing exactly due to the above mentioned properties, it has been neglected because of its technical difficulties. Existence is quite often a problem, especially when preferences can exhibit cycles, whereas uniqueness is even more difficult to achieve. It is critical to point out that a particular outcome may be dimmed stable given a certain solution set, and unstable given another solution set. Uniqueness of the solution set is thus, not an immediate characteristic. The possible multiplicity of solution sets is intuitively explained by the fact that different sets of proposals may be considered during a negotiation process. and this different set of proposals can be depicted by different solution sets.

Within the context of cartel formation we consider a set $\sigma$ which is a collection of all stable cartels, i.e., $\sigma=\left\{C_{k}, C_{h}, \ldots, C_{l}\right\}$. A cartel $C_{k}$ is stable given $\sigma$ and thus. $C_{k} \in \sigma$ if it is both internally and externally stable given $\sigma$.

A cartel is considered to be internally stable if, loosely speaking, no firm wishes to exit it. So far, a firm compared its current profits $\pi^{c}(k)$ with the profits of the fringe it would join $\pi^{f}(k-1)$. We claim that such a comparison is justified only if $C_{k-1}$ is a stable cartel, i.e., $C_{k-1} \in \sigma$ as well, which would imply that if $C_{k-1}$ becomes the status quo it would remain so. Otherwise, if $C_{k-1} \notin \sigma$ once at $C_{k-1}$ some other agent may wish to either join it or exit it. Thus, the very first agent when contemplating whether to exit $C_{k}$ or not he should compare it to final stable outcome that will rise. More formally, 
Definition $2 A$ cartel $C_{k}$ is internally stable given $\sigma$ if there does not exist a finite sequence of cartels $C_{k-1}, C_{k-2}, \ldots C_{k-j}, \ldots, C_{k-m}$, where $m \in\{1,2, \ldots, k\}$ such that $C_{k-m} \in \sigma$ and $\pi^{c}(k-j)<\pi^{f}(k-m)$ for every $j=0,1, \ldots, m-1$.

A parallel process describes external stability. A cartel $C_{k}$ is externally stable if (loosely speaking) no firm wishes to join it. Again the firm makes such a decision by comparing its profits under the status quo $\pi^{f}(k)$ with the profits it will make once it joins, namely $\pi^{\mathfrak{c}}(k+1)$. Such a comparison is justified only if $C_{k+1} \in \sigma$ is a stable cartel itself and thus, no more agents wish to enter or exit. The firm should compare its status quo with the final outcome that will arise. Formally,

Definition 3 A cartel $C_{k}$ is externally stable given $\sigma$ if there does not exist a finite sequence of cartels $C_{k+1}, C_{k+2}, \ldots C_{k+j}, \ldots, C_{k+m}$, where $m \in\{1,2, \ldots, n-k\}$. such that $C_{k+m} \in \sigma$ and $\pi^{c}(k+m)>\pi^{f}(k+j)$ for every $j=0,1, \ldots, m-1$.

Finally, a cartel $C_{k} \notin \sigma$ is excluded from our solution set if it is not stable and therefore either its internal or external stability is violated. Conclusively:

Definition 4 A cartel $C_{k}$ is stable given $\sigma$ if it is both internally and externally stable given $\sigma$. A set of cartels $\sigma$ is stable if every cartel $C_{k} \in \sigma$ is stable given $\sigma$ and if every cartel $C_{k} \notin \sigma$ is either internally or externally unstable given $\sigma$.

Note that the null cartel $C_{0}$ that contains no firms is trivially internally stable since there does not exist a firm to exit, and that a perfectly collusive market where the cartel $C_{n}$ contains all the firms is trivially externally stable, since there do not exist more firms to join. When a cartel $C_{k}$ is stable we characterize its fringe $F_{k}$ as stable as well. 
It is evident by now that our solution set $\sigma$ is built in the spirit of the vN-M stable set by allowing for no inner contradiction and accounting for every exclusion. We have, however, modified the dominance relation by allowing for indirect dominance in the spirit of Harsanyi.

We mentioned earlier that one of the major drawbacks of the stable set is its difficulty to exist. Yet, we were able to establish an existence result for our model with only one condition. The requirement for existence is that the profits of the fringe increase as its size shrinks. It is worth mentioning that such a requirement is not unnatural, in fact, the following section (Linear Case) describes a situation where this condition always holds. To see the mildness of this requirement consider the extreme case where the market is perfectly competitive, the usual conclusion under "normal" conditions is that profits are zero. Now consider the case were all the firms collude and behave like a monopolist, again the usual conclusion is that the firms make positive profits and each firm has an incentive to deviate because the fringe makes even higher profits. Therefore, as the size of the fringe shrunk from $n$ to 0 its profits increased. We could have imposed some additional sufficient conditions on the cost function that could guarantee this property, but we found such an approach confiding, since it may very well be true besides such conditions, we are leaving it up to the interested reader to examine whether the model he is considering satisfies our condition.

Theorem 5 With $n$-finite, if $\pi^{f}$ increases as $k$ increases, there always exists a nonempty set of stable cartels, $\sigma$.

Proof. In order to proceed with the proof we need to recall first the following results that were established by $D^{\prime}$ Aspremont et al.(1983): 
(1) For $k \geq 1, \pi^{e}$ increases as $k$ increases and

(2) For $n$-finite there exists $k \in\{1, \ldots, n\}$ such that $\pi^{c}(k) \geq \pi^{f}(k-1)$ and $\pi^{c}(k+$ 1) $\leq \pi^{f}(k)$.

We will construct a stable set $\sigma$ for the above problem. Consider $C_{n}$, if $\pi^{c}(n)>$ $\pi^{f}(n-1)$ then $\sigma=\left\{C_{n}\right\}$.

If $\pi^{c}(n)<\pi^{f}(n-1)$ then we continue examining smaller cartels by decreasing the size by one firm at a time until we reach cartel $C_{k_{1}}$ such that $\pi^{e}\left(k_{1}\right)>\pi^{f}\left(k_{1}-1\right)$. From D' Aspremont et al's result (2) it is obvious that such a $k_{1}$ exists. We include $C_{k_{l}}$ in $\sigma$. Now, we start going up again (increasing the size of cartels by one at a time) until we reach $C_{k_{2}}$ where $k_{2}>k_{1}$ and $\pi^{c}\left(k_{2}\right)>\pi^{f}\left(k_{1}\right)$. In fact, if such a cartel $C_{k_{2}}$ exists, it does not immediately succeed $C_{k_{1}}$, that is $k_{2}>k_{1}+1$, otherwise $\pi^{2}\left(k_{2}\right)>\pi^{f}\left(k_{1}\right) \Leftrightarrow \pi^{c}\left(k_{2}\right)>\pi^{f}\left(k_{2}-1\right)$ which would contradict the original finding of $C_{k_{1}}$-during the original search for $C_{k_{1}}$ we should have stopped at $C_{k_{2}}=C_{k_{1}+1}$ instead. If such a cartel of size $k_{2}$ exists, we include it in $\sigma$ and continue going up (increasing the size of cartel by one at a time) until we reach $C_{k_{3}}$ where $k_{3}>k_{2}+1$ and $\pi^{\mathcal{c}}\left(k_{3}\right)>\pi^{f}\left(k_{2}\right)$. If such a cartel of size $k_{3}$ exists, we include it in $\sigma$. We continue in this manner until we have reached $C_{n}$. We now have a set $\sigma=\left\{C_{k_{1}}, C_{k_{2}}, \ldots, C_{k_{1}}, \ldots, C_{k_{m}}\right\}$ for $j=1,2, \ldots, m$ where $m \leq n, k_{j}>k_{(j-1)}+1$ and $k_{m} \leq n$. To summarize the construction of $\sigma$, we know that $\pi^{c}\left(k_{j}\right)<\pi^{f}\left(k_{j}-1\right)$ and that $\pi^{c}\left(k_{j}\right)>\pi^{f}\left(k_{j-1}\right)$ for $j>1$ whereas $\pi^{c}\left(k_{1}\right)>\pi^{f}(k-1)$ for every $k \leq k_{1}$. Note that $\sigma \neq \emptyset$ since, as argued earlier, we can always find at least $C_{k_{\mathrm{T}}}$. Also note that for $m=1$ and $k_{m}=n$ we have the special case of $\sigma=\left\{C_{n}\right\}$.

Now we will argue that $\sigma$ is stable. Firstly we will show that all the elements inside $\sigma$ are both internally and externally stable. And then we will show that those 
excluded from $\sigma$ are accounted for. If $|\sigma|=1$, that is, it is a singleton then the one cartel in it is trivially both internally and externally stable since firms have essentially no other alternative, no other stable cartel, smaller or larger to want to deviate to.

If $|\sigma|>1$, that is $\sigma$ contains more than one cartel we start by considering $C_{k_{1}}$. It is internally stable since no smaller stable cartel belongs to $\sigma$, therefore firms have nowhere (stable) to go if they exit the cartel. It is externally stable because even though $\pi^{c}\left(k_{2}\right)>\pi^{f}\left(k_{1}\right)$ by the construction of $\sigma$, at the last step of the sequence $\left(F_{k_{2}-1}\right)$ that would lead us from $F_{k_{1}}$ to $C_{k_{2}}$ no agent wishes to join $C_{k_{2}}$ since $\pi^{c}\left(k_{j}\right)<$ $\pi^{f}\left(k_{j}-1\right)$ for every $j>1$, thus for $j=2$ we have $\pi^{c}\left(k_{2}\right)<\pi^{f}\left(k_{2}-1\right)$.

Now let us consider some $C_{k_{j}}$, where $1<j<m$. It is internally stable because $\pi^{c}\left(k_{j}\right)>\pi^{f}\left(k_{j-1}\right)$ while $\pi^{c}\left(k_{j}-1\right)<\pi^{f}\left(k_{j-1}\right), \ldots, \pi^{c}\left(k_{j}-l\right)<\pi^{f}\left(k_{j-1}\right), \ldots . \pi^{c}\left(k_{j-1}+\right.$ 1) $<\pi^{f}\left(k_{j-1}\right)$ for $l=1,2, \ldots,\left(k_{j}-k_{j-l}-1\right)$. If a firm, member of $C_{k_{j}}$, exits its cartel it induces $C_{k_{j}-1}$, by joining $F_{k_{j}-1}$, Nevertheless, once $C_{k_{j}-1}$ is induced, another firm will join the fringe of $C_{k_{j}-2}$, namely $F_{k_{j}-2}$, and then another firm will join the fringe $F_{k_{3}-3}$, and so on until they reach $C_{k_{(j-1)}+1}$, where the last firm will join the fringe of the following cartel, $C_{k_{(j-1)}}$ since they all prefer the final outcome $F_{k_{j-1}}$ to their current cartel $C_{k_{j}-l}$ except the very first firm that exited $C_{k_{j}}$. Note that $F_{k_{j-1}}$ is the terminal outcome since $C_{k_{j-1}}$ is a stable outcome by assumption and therefore internally stable, thus no more firms would want to deviate and join the fringe $F_{k_{(j-1)}-1}$.

$C_{k_{j}}$ is externally stable since $\pi^{c}\left(k_{j+1}\right)<\pi^{f}\left(k_{j+1}-1\right)$ even though $\pi^{c}\left(k_{j+1}\right)>$ $\pi^{f}\left(k_{j}\right)$. That is, although members of the fringe of $C_{k_{j}}$, namely $F_{k_{j}}$ would like to join the cartel $C_{k_{(j+1)}}$ since they prefer it to their current situation $F_{k_{j}}$, at the last step of the sequence that would lead to $C_{k_{(j+1)}}$, namely at $F_{k_{(j+1)}-1}$ no more members of this 
fringe wish to join the cartel $C_{k_{(j+1)}}$.

Lastly consider $C_{k_{m}}$, which is externally stable since there is no other larger stable cartel for the fringe members to want to join. If $k_{m}=n$ then the cartel is trivially externally stable since everyone has joined already. $C_{k_{m}}$ is internaily stable for the same reason $C_{k_{j}}$ is, that is, because $\pi^{c}\left(k_{m}\right)>\pi^{f}\left(k_{m-1}\right)$ and $\pi^{c}\left(k_{m}-1\right)<\pi^{f}\left(k_{m-1}\right), \ldots, \pi^{c}\left(k_{m}-l\right)<\pi^{f}\left(k_{m-1}\right), \ldots, \pi^{c}\left(k_{m-1}+1\right)<\pi^{f}\left(k_{m-1}\right)$ for $l=1,2, \ldots,\left(k_{m}-k_{m-l}-1\right)$.

Now we will argue that every $C_{h} \notin \sigma$ is accounted for, that is either its internal or external stability is violated. If $k_{\mathrm{l}}>1$, we consider $C_{h}$, where $0 \leq h<k_{\mathrm{l}}$. Since by construction of $\sigma, \pi^{c}\left(k_{1}\right)>\pi^{f}\left(k_{\mathrm{L}}-1\right)$ and by assumption the profits of the fringe decrease as the fringe grows, it is the case that $\pi^{c}\left(k_{1}\right)>\pi^{f}\left(k_{1}-1\right)>\ldots>\pi^{f}(h)>$ $\ldots>\pi^{f}(0)$, thus cartels $C_{1}, \ldots, C_{h}, \ldots, C_{k_{2}-\mathrm{l}}$ are externally unstable since members of their fringes want to join them and reach $C_{k_{1}}$.

If $k_{m}<n$, we consider $C_{h}$ where $k_{m}<h \leq n$. By construction of $\sigma$ we know that $\pi^{c}(n)<\pi^{f}\left(k_{m}\right), \ldots, \pi^{c}(h)<\pi^{f}\left(k_{m}\right), \ldots, \pi^{c}\left(k_{m}+1\right)<\pi^{f}\left(k_{m}\right)$, thus cartels $C_{n}, C_{n-1}, \ldots, C_{h}, \ldots, C_{k_{m}+1}$ are internally unstable since their members wish to join the fringe $F_{k_{m}}$.

If $|\sigma|>1$, that is, it contains more than one cartel, consider $C_{h}$ where $k_{j}<$ $h<k_{(j+1)}$. By construction of $\sigma$ we know that $\pi^{c}\left(k_{j+1}-1\right)<\pi^{f}\left(k_{j}\right), \ldots, \pi^{c}(h)<$ $\pi^{f}\left(k_{j}\right), \ldots, \pi^{e}\left(k_{j}+1\right)<\pi^{f}\left(k_{j}\right)$, thus cartels $C_{k_{(j+1)}-1}, \ldots, C_{h}, \ldots, C_{k_{j}+1}$ are internally unstable since their members wish to join the fringe $F_{k_{j}}$. 


\section{Linear Case}

This section studies the specific case of a linear demand and marginal cost functions, in order to primarily illustrate that the requirement for existence is always satisfied in this special case, and secondarily to provide some additional insight to the mechanics of the model.

We consider a linear demand function $Q=a-b P$, where $a>0, b>0$ and $Q=$ $\sum_{i \in N} q^{i}$ is the total market quantity. We can drop the superscript $i$ on individual quantities since all firms are identical; we will just differentiate between cartel output $q^{f}$ and fringe output $q^{f}$. The total cost function is $C(q)=\frac{\mathrm{L}}{2} c q^{2+}$, where $c>0$.

As described in the general case, the fringe behaves competitively by setting its marginal cost $C^{\prime}(q)=c q^{f}$ equal to the price determined by the cartel. i.e.. $c q^{j}=$ $P \Leftrightarrow q^{f}=\frac{1}{c} P$. Thus, the fringe as a whole produces $Q^{f}=(n-k) q^{f}=\frac{n-k}{c} P$

The residual demand faced by the cartel is given by

$$
R D=Q-Q^{f}=(a-b P)-\frac{n-k}{c} P=a-\left(\frac{b c+n-k}{c}\right) P
$$

The cartel determines how much output to produce by setting its marginal revenue (as derived from the residual demand) equal to its marginal cost. From straight forward calculations we have the individual cartel output:

$$
q^{c}=\frac{a}{b c+n+k}
$$

The market price is determined from the residual demand:

$$
P=\frac{a c(b c+n)}{(b c+n)^{2}-k^{2}} .
$$

This case differs from the one appearing in Donsimoni et al. (1986) in that their cost function is $C\left(q_{i}\right)=\left(\frac{1}{2 c}\right) q_{i}^{2}$ 
Each fringe firm then produces:

$$
q^{f}=\frac{a(b c+n)}{(b c+n)^{2}-k^{2}}
$$

Lastly, the respective profits are:

$$
\begin{aligned}
\pi^{c}(k) & =\frac{a^{2} c}{2\left[(b c+n)^{2}-k^{2}\right]} \\
\pi^{f}(k) & =\frac{a^{2} c(b c+n)^{2}}{2\left[(b c+n)^{2}-k^{2}\right]^{2}}
\end{aligned}
$$

It is easy to see that $\frac{\partial \pi^{f}}{\partial k}>0$ is trivially satisfied. Therefore, in the linear we can always find a non-empty stable solution set $\sigma$, and thus a stable cartel.

\section{Conclusion}

What we attempted to accomplish through this project is to encompass foresight and consistency. We argued that cartel stability is analyzed, so far, by imposing myopia on the firm's behavior, and unless this is a deliberate choice aiming to describe some particular aspect of the agent, it is an assumption that should be avoided.

In this spirit, we employed a modified version of the notion of $\mathrm{vN}-\mathrm{M}$ stability to analyze oligopolistic markets. The stable set in conjunction with indirect dominance (in Harsanyi's spirit) provided us with a solution concept that embodies the desired features. Namely, agents foresee finitely many steps ahead and make their decisions by comparing the final stable outcomes of their actions. We were able to prove existence of a set of stable cartels under an assumption concerning the monotonicity of the fringe profits. An immediate corollary is that in the case of linear demand and marginal cost fimctions there always exists a set of stable cartels. Uniqueness of the set of stable cartels was not examined in this paper, and it is still an open question. Notice the difference between uniqueness of a stable cartel and uniqueness of a set 
of stable cartels. The former, is obviously not true in the example we presented in the introduction, were we claim that two cartels are stable, yet this does not imply that under some severe conditions on the relationship of the demand and cost functions uniqueness of cartel stability may be achieved. The latter though, is a far more complex and desirable property. Uniqueness of the set of stable cartels, loosely speaking, will not require as harsh restrictions as uniqueness of stable cartel, for it to be true. Finally, uniqueness of cartel stability although technically attractive, intuitively seems rather strong and unrealistic.

Nonetheless, foresight is integrated in our model where the cartel behaves as a price leader and the fringe behaves competitively. Our solution concept could very well be applied to different models were the fringe behaves a la Cournot. or where the product is not homogeneous, or were the firms are not identical, etc. But most importantly the assumption we believe should be the first to be relaxed is that of purely non-cooperative behavior embodied in our model. It is explicit that each firm moves independently, and does not, ever, consider colluding with others, which is indeed an antithesis. The cartel is permitted to acknowledge and enjoy the benefits of collusive behavior, but once a member exits the cartel he forgets all about it. We are not implying that collusive behavior should become axiomatic and therefore insist upon binding agreements. On the contrary, we still stand by the presumption that any coalition should be immune to credible deviations, by individuals or other coalitions, which are immune themselves, etc., but as long as it is, it should be allowed to form. Links between the various price leadership models and the purely cooperative approaches through coalition structures is another venue pending investigation.

Last but not least, the tools The Theory of Social Situations, Greenberg (1990) 
has to offer should be exploited. Admittedly, one of the difficulties in analyzing cartel stability is the inability to find a way (methodology) to describe the various models accurately and explicitly, that is, cooperative vs non-cooperative, dynamic versus static. It seems to be the case that most theorists would cast their oligopolistic market somewhere in the middle. The diversity TOSS allows for may be exactly what we need to formalize cartel formation.

\section{References}

I. D'Aspremont and Jaskold-Gabsewicz J. (1986). "On the stability of Collusion," in New Developments in the Analysis of Market Structure. (Stiglitz, J.E. and Mathewson C.F. Eds.) MIIT Press.

2. D'Aspremont. C., JaCQUEmin A., Jaskold-GabseWiCz J. AND WeyMARK J. (1983). "On the Stability of Collusive Price Leadership." Canadian Journal of Economics 16, 17-25.

3. Aumann, R. J., And Dreze, J. H. (1974). "Cooperative Games with Coalition Structures," International Journal of Game Theory 3, 217-237.

4. Chwe, M. S.-Y. (1994). "Farsighted Coalitional Stability," Journal of Economic Theory 63, 299-325.

5. DonstMoni, M.-P. (1985). "Stable Heterogenous Cartels," International Journal of Industrial Organization 3, 451-467.

6. Donstmont, M.-P., Economides N. S., and Polemarchakts H. M. (1986). "Stable Cartels," International Economic Review 27, 317-327. 
7. GreenberG, J. (1990). The Theory of Social Situations: An Alternative Game-Theoretic Approach. Cambridge University Press.

8. Greenberg, J. (1994). "Coalition Structures," in Handbook of Game Theory, (R. Aumann and S. Hart, Eds.) Amsterdam: Elsevier.

9. HARSANYI, J.C. (197t). "Interpretation of Stable Sets and a Proposed Alternative Definition," Management Science 20, 1472-1495.

10. Johansen, L. (1977). "Price-Taking Behavior," Econometrica 45, 1651-1656.

11. LI, S. (1992). "Far-sighted Strong Equilibrium and Oligopoly," Economics Letters 40, 39-44.

12. Martin, S. (1993). Advanced Industrial Economics. Basil Blackwell.

13. Postlewait, A. AND Roberts, J. (1976). "The Incentives of Price Taking Behavior in Large Exchange Economies," Econometrica 44, 115-128.

14. _.... AND _ (1977). "A note on the stability of large cartels," Econometrica $45,1877-8$.

15. Prokop, J. (1999). "Process of Dominant-Cartel Formation," International Joumal of Industrial Organization 17, 241-257.

16. Selten, R. (1973). "A Simple Model of Imperfect Competition, where 4 are Few and 6 are Many," International Journal of Game Theory 2, 141-201.

17. Shaffer, S. (1995). "Stable Cartels with a Cournot Fringe," Southern Economic Journal 61, 744754. 
18. Thoron, S. (1998). "Formation of a Coalition-Proof Stable Cartel," Canadian Journal of Economics 31, 63-76.

19. XUE, L. (1998). "Coalitional Stability under Perfect Foresight," Economic Theory 11 603-627.

20. von NeUmanN, J. AND MoRgENSTERN, O. (1944). Theory of Games and Economic Behavior. Princeton University Press 